\title{
Dynamic Scheduler Management Using Deep Learning
}

\author{
James Hall, Klaus Moessner, Richard Mackenzie, Francois Carrez and Chuan Heng Foh
}

\begin{abstract}
The ability to manage the distributed functionality of large multi-vendor networks will be an important step towards ultra-dense $5 G$ networks. Managing distributed scheduling functionality is particularly important, due to its influence over inter-cell interference and the lack of standardization for schedulers. In this paper, we formulate a method of managing distributed scheduling methods across a small cluster of cells by dynamically selecting schedulers to be implemented at each cell. We use deep reinforcement learning methods to identify suitable joint scheduling policies, based on the current state of the network observed from data already available in the RAN. Additionally, we also explore three methods of training the deep reinforcement learning based dynamic scheduler selection system. We compare the performance of these training methods in a simulated environment against each other, as well as homogeneous scheduler deployment scenarios, where each cell in the network uses the same type of scheduler. We show that, by using deep reinforcement learning, the dynamic scheduler selection system is able to identify scheduler distributions that increase the number of users that achieve their quality of service requirements in up to $77 \%$ of the simulated scenarios when compared to homogeneous scheduler deployment scenarios.
\end{abstract}

Index Terms-Reinforcement Learning, Deep Learning, Scheduling

\section{INTRODUCTION}

$\mathbf{T}$ HE scheduler, located in the medium access control (MAC) layer of the radio access network (RAN), is responsible for assigning the spectral resources of the air interface to the users in the network. Spectral resources are divided into spectral-temporal resources blocks, which are individually assigned to users by the scheduler. The control that the scheduler has over the usage of resources in the RAN influences the high level performance metrics used to assess the network, including throughput, fairness and latency. These

This work was supported in part by the U.K. Engineering and Physical Science Research Council and in part by BT Applied Research through an Industrial Cooperative Awards in the Science and Technology Studentship. We would also like to acknowledge the support of the University of Surrey 5GIC (http://www.surrey.ac.uk/5gic) members for this work. The work of F. $\mathrm{J}$. The views expressed here are those of the authors and do not necessarily reflect those of the affiliated organizations.

J. Hall, K. Moessner, F. Carrez and C. H. Foh are with 5GIC, the Institute of Communication Systems, University of Surrey, Guildford, GU2 7XH, U.K. Email: \{j.e.hall, k.moessner, f.carrez, c.foh\}@ surrey.ac.uk

R. Mackenzie is with BT Technology, Adastral Park, Ipswich, UK. Email: richard.mackenzie@bt.com

K. Moessner also with Professorship Communications Engineering, Faculty of Electrical Engineering and Information Technology, Technical University Chemnitz, Germany. indicators are monitored because of their influence on the quality of service provided to users in the network.

A large body of literature exists around scheduling methods for the LTE network architecture. These methods are evaluated by a number of surveys of the most commonly used scheduling methods for LTE networks, highlighting the key themes and the limitations imposed by the network architecture [1], [2]. Scheduling is performed in a distributed manner, where each cell site in a network performs its scheduling independently. The distributed methods examined all belong to one of three categories [2]: channel unaware, where scheduling decisions are made independent to the network environment; channel aware, where the scheduling decision is made in a manner that is aware of the channel conditions of its users, ultimately making better use of the network resources; and quality of service (QoS) aware, whereby the scheduler is aware of the channel quality and QoS constraints of its users.

For future networks e.g. 5G, new approaches to the scheduling process could be deployed in conjunction with these traditional methods. Many of these new methods focus, in particular, on addressing the problem of inter-cell interference. With increased network density and higher throughput requirements, inter-cell interference influences key performance measures of the network Hossain et al. [3]. So far, network architectures have used distributed scheduling methods. With the introduction of $5 \mathrm{G}$ and its cloud radio access network (CRAN), centralized scheduling also becomes possible.

Performing scheduling for multiple cells simultaneously, from a central entity, allows greater control over inter-cell interference experienced by users. This form of coordinated scheduling is particularly well suited to CRAN deployments, where the functional split between the central unit and remote radio heads allows for a centralised MAC [4]. Douik et al. [5] uses scheduling in a centralised manner over a cluster of cells, allowing for an optimal resource allocation. However, as described by Nardini et al. [6], the computation required to solve the scheduling problem in an optimal manner is prohibitively large, limiting this method to be applied only to small clusters of cells. However, only a subset of CRAN architectures supports centralised scheduling, particularly functional split options 5-8 explored by 3GPP [4].

Much of the future 5G network environment could consist of architectures that do not support centralised scheduling methods, and instead will continue to rely on distributed scheduling methods. In particular, this is the case for small cells, which are expected to be deployed in the highest densities, often in an unplanned manner.

Nardini et al. [6] present a method that performs scheduling 
in a semi-distributed manner in networks that contain tens of cells. The central entity provides allocation masks that dictate to the cells which resources the distributed scheduling methods can allocate. Ramos et al. [7] present a decentralised method where cells can identify which resource blocks are assigned to users experiencing high interference and communicate this over the X2 interface to their neighbours. Neighbouring cells then avoid using those resources when scheduling their own users. These semi-distributed methods of resource scheduling solve the scalability and architecture problems of centralised scheduling. However, due to their cooperative nature, clusters of cells operating such methods are required to deploy the methods ubiquitously.

A growing body of literature looks to schedule users in a more conservative manner, such that the inter-cell interference produced by the network equipment is reduced. In LópezPérez et al. [8], resource allocation is performed by minimising the power usage of each cell, under the condition that each user's service requirement is fulfilled. This type of scheduler, known as margin adaptive [9], has been shown to converge to frequently reuse patterns that dynamically change to account for network conditions [10]. However, these methods formulate scheduling as an optimisation problem, where finding optimal solutions cannot be performed at a suitable time scale for real-time user scheduling. Instead, implementations of these types of scheduler require heuristic algorithms or approximate optimization techniques to solve, meaning the solution found is often suboptimal. Further, users under these scheduling conditions cannot exceed their throughput requirement, limiting the Quality of Experience (QoE) offered to these users, and the ability to fulfil the QoS requirements of these methods is dependent on the capacity of the cell. For these reasons, the suitability of margin adaptive scheduling is subject to the network conditions. Specifically, these methods have been proposed prominently for femto-cells, where the channel bandwidth and volume of users is relatively low and extensive network planning is unfeasible [11].

With a large and diverse range of scheduling methods available, many of which cater for a predictable set of network and traffic conditions, it can be difficult to select suitable scheduling methods for use across the network. Further, it has been observed that traffic conditions in cellular networks experience large spatio-temporal variations. For example, Chen et al. [12] observe these variations in macro-cell networks. These variations can cause the suitability of scheduling methods to fluctuate over the course of hours, days or weeks, compounding the difficulty in selecting schedulers.

Managing network functionality, such as scheduling methods over large networks, can be a challenge, even for wellresourced operators. To counter this, many studies propose artificial intelligence, and in particular, machine learning, as technologies capable of resolving these management tasks autonomously [13], [14]. Some groups, such as O-RAN, propose the inclusion of intelligent network management functions directly into the RAN architecture [15], facilitating both nonreal-time and near-real-time RAN control based on state-ofthe-art machine learning techniques.

With the aim of autonomous network control, reinforcement learning is one area of machine learning that has seen great focus in network management problems. Reinforcement learning is used in network control problems, as it allows the creation of agents that are able to learn optimal or near optimal control policies autonomously. As such, reinforcement learning has been proposed for a range of problems and new applications within the mobile network [16]. For example, Jaber et al. [17] applied reinforcement learning on a range extension coefficient of small cells to optimize the quality of the backhaul links.

Deep reinforcement learning is a development of traditional reinforcement learning methods by introducing deep neural networks used to generalise an action value function or policy functions over a large state space [18]. The feature extraction ability of deep reinforcement learning improves traditional reinforcement learning methods for low level control problems. For example, Chinchali et al. [19] use deep reinforcement learning to control the amount of IoT traffic that can be scheduled, depending on the congestion on the network. The ability to generalise over a large state space makes these techniques well suited to high-level management problems. A survey by Zhang et al. [20] summarises a range of applications of these techniques.

In commercial networks, the operator will usually have a scheduler selection decision to make when configuring a base station. Typically, a vendor will provide more than one scheduler option, for example, a choice of either a proportional fair scheduler, round robin, maximum throughput or a maxmin scheduler. In the past, an operator could make this decision as part of the regular network planning activities. For future high density deployments (such as for $5 \mathrm{G}$ networks), a more dynamic solution to the scheduler selection problem is required, one that can respond to greater inter-cell interference, unplanned deployment, and multi-vendor clusters.

In this paper, we propose a new approach to manage distributed scheduling methods autonomously across multiple cells simultaneously, reducing the requirement for cell planning and increasing the flexibility of scheduling methods in the network. A distributed approach to scheduler management is proposed by Comşa et al. [21], where a flexible scheduler uses interchangeable scheduling policies to best serve the QoS requirements of its users. To select from these policies, the authors suggest a reinforcement learning framework, where the QoS offered by each scheduling policy is estimated by an individual neural network. In our work we look to perform scheduler management jointly over a group of cells rather than on a per cell basis individually allowing our method to take advantage of schedulers that provide neighbouring cells with beneficial interference conditions. To account for the increased problem space we propose the use of deep reinforcement learning methods that provide generalisation over the state space.

In our method, all resource scheduling is performed in a distributed manner by scheduling methods ( [22], [23]). A central entity is responsible for managing these distributed scheduling methods through dynamic scheduler selection. We propose the use of reinforcement learning and nature-inspired algorithms to perform scheduler selection at this central entity. This method facilitates the deployment of scheduling methods 
that are well suited to situations where the network conditions fall within specific constraints. We propose two schemes for scheduler selection to solve the scheduler selection problem; the first uses nature-inspired optimization algorithms while the second uses deep reinforcement learning. In Section III the system model is described and the problem will be formulated. In Section III the proposed schemes are detailed. In Section IV and $\mathrm{V}$ simulation based evaluation of the proposed methods are detailed and reported on. These simulations compare the performance of our scheduler selection strategies with static homogeneous strategies that are representative of current network deployment methods.

\section{SySTEM MODEL}

In this study, a cellular network is considered, consisting of $\mathcal{N}_{c}$ cells serving $\mathcal{N}_{u}$ UEs distributed within the coverage area of the cells. Each user can be served by a single cell in the network. For each downlink channel, orthogonal frequency division multiple access (OFDMA) is assumed. In OFDMA, a single channel is formed of a large number of orthogonal sub-channels. Additionally, the channel is divided in the time domain into a number of 1 transmission time interval (TTI) sub-frames. In our model, it is assumed that each cell that is responsible for scheduling is associated with users on the OFDMA channel. Each cell is able to schedule users to any of the resource blocks on the channel and the transmit power of the cell is divided equally among all the resource blocks in the grid.

Each cell in the network can perform scheduling according to one of two predefined scheduling methods from scheduler set $\mathcal{S}_{c} \in\{0,1\}$ where $\mathcal{S}_{c}=0$ corresponds to a proportional fair scheduler [22] and $S_{c}=1$ corresponds to a rate guaranteed max-min scheduler [23]. A central entity is responsible for selecting the active schedulers from scheduler set $S_{c}$ in each of the cells in the network.

The proportional fair scheduler assigns each resource block greedily according to a fairness metric which weights the quality of the user channels against the throughput that has been provided to the users over a window of frames. The QoS guaranteed max-min scheduler maximises the service offered to the worst served user, in doing so the max-min scheduler offers similar service to all users. Additionally, the QoS guarantees that where users have matched their QoS guarantees, the user is scheduled no further resources.

Consider that each user in the network has a service throughput requirement $T P^{(r e q)}$ and allow $\lambda_{i} \in\{0,1\}$, to indicate user satisfaction according to:

$$
\lambda_{i}= \begin{cases}1 & \text { if } T P_{i} \geq T P^{(r e q)} \\ 0 & \text { otherwise }\end{cases}
$$

where $\lambda_{i}$ is an indicator function whose value is 1 if the user $i$ is satisfied and 0 if not, and $T P_{i}$ is the throughput received by user $i$. The overall satisfaction rate $\mathcal{U}$ is defined by:

$$
\mathcal{U}=\frac{\sum_{i=1}^{\mathcal{N}_{u}} \lambda_{i}}{\mathcal{N}_{u}}
$$

Frequency reuse of 1 is assumed for all cells meaning inter cell interference impacts signal quality of users in neighbouring cells. We therefore define a joint scheduling policy as $\sigma=<$ $s_{1}, s_{2}, \ldots, s_{N c}>$ where $s_{i} \in S_{c}$. The satisfaction rate for a joint scheduling policy is defined as:

$$
\mathcal{U}_{\sigma}=\frac{\sum_{i=1}^{\mathcal{N}_{u}} \lambda_{i, \sigma}}{\mathcal{N}_{u}}
$$

where $U_{\sigma}$ is the satisfaction rate offered by joint scheduling policy $\sigma$, and $\lambda_{i, \sigma}$ is an indicator function that denotes whether user $i$ is satisfied under joint scheduling policy $\sigma$. Finally, the dynamic scheduler selection problem (DSS) can be defined, where the central entity seeks to select the joint scheduling policy $\sigma$ that achieves the largest satisfaction rate across the entire network:

$$
\max _{\sigma} \mathcal{U}_{\sigma}
$$

The schedulers chosen for this study are selected for their contrasting effects on the satisfaction rate defined in Eq. (2). The QoS guaranteed scheduler attempts to simultaneously satisfy all users by maximising the throughput offered to the worst served user. When there is adequate resource, all users can be satisfied. However, when resources are insufficient, each user may receive some amount of resources, but the amount may not satisfy user requirement.

Conversely, the fairness metric used by the proportional fair scheduler ensures that users with better quality channels prioritised. Consequently, when resources are insufficient to satisfy all users, the users with the best channels will still be satisfied. However, when resources are sufficient to satisfy all users, the users with the worst channels may be unsatisfied.

\section{DynAmic Scheduler SElEction}

In this section we outline our approaches for solving the dynamic scheduler selection problem using a central entity and nature-inspired algorithms. We propose two approaches; the first uses genetic algorithm to dynamically converge to a solution. The second approach uses deep reinforcement learning to learn the networks response to the joint scheduling problem over a large time scale. We also consider that the DSS schemes presented in this study make periodic decisions on which joint scheduling policy is implemented.

\section{A. Genetic Algorithm Dynamic Scheduler Selection}

In this method we use a genetic algorithm, i.e. a natureinspired optimization algorithm, to make joint scheduling policy decisions for the network. The genetic algorithm is a threestage (fitness, selection and mutation) iterative optimization algorithm that uses a utility, which in this case refers to the satisfaction rate received, to influence the next generation. In a genetic algorithm a population $p$ undergoes the fitness, selection and mutation stages, and each chromosome in the population represents a joint scheduling policy.

In the fitness stage, the fitness, or satisfaction rate, of each chromosome to the DSS problem is evaluated through implementation in the network, where the fitness of the chromosome $q$ is: 


$$
f_{i t}=\mathcal{U}_{\sigma}
$$

Once all the chromosomes in a population have been evaluated, a new generation of that population is generated according to the fitness recorded from the previous generation. The new generation of chromosomes is created using the final two stages of the genetic algorithm: Selection and Mutation. In the selection stage, two parents are selected from the previous generation. The value of each gene of the new chromosome is randomly selected from the values of the parents corresponding genes. Parents are selected using fitness proportional selection.

Finally, the new generation undergoes mutation where the values in the new generation are changed with probability $\mu$. The mutation stage encourages additional exploration of the problem space by introducing values not present in the previous generation.

Evaluating fitness of chromosomes over changing network conditions results in variations in $f i t_{q}$ such that consecutive periods of implementation of the same joint scheduling policy $\sigma$ result in different satisfaction rates. To reduce the impact of network variations on the solutions found using genetic algorithms, and to prevent overfitting based on favourable network conditions, the selection stage of the algorithm is altered to account for this. We set the fitness of each unique chromosome in the population is equal to the minimum fitness of all identical chromosomes.

\section{B. Reinforcement Learning Dynamic Scheduler Selection}

Reinforcement learning allows the implementation of agents that learn how actions or sequences of actions are rewarded through interactions with the environment. For the DSS problem, the reinforcement learning agent will learn the expected satisfaction rate (reward) for implementing a joint scheduling policy, given the observed state of the environment. For this study the DSS problem is formulated as a contextual multiarmed bandit problem; in these problems the aim of the agent is to maximize the reward it receives. The agent's decisions are contextualized by information about the state of the network (environment).

The reinforcement learning dynamic scheduler selection (RL-DSS) agent observes the volume of traffic and the quality of channels in the network, and instantaneously selects a joint scheduling policy $\sigma$ that fulfils (4). Users periodically report the quality of the channels in the format of Channel Quality Indicators (CQI). Based on [24], CQI values are reported in the range 1-15. The value reported corresponds to the highest modulation coding scheme (MCS) that the user can decode with a maximum of $10 \%$ error rate. A single CQI value can be reported across the entire channel (wideband CQI) or on a per sub-band basis (sub-band CQI). The CQI reports are collated into a state matrix defined by:

$$
\mathcal{X}=\left[\begin{array}{ccccr}
x_{1,1} & x_{1,2} & x_{1,3} & \ldots & x_{1, N} \\
x_{2,1} & x_{2,2} & x_{2,3} & \ldots & x_{2, N} \\
\ldots \ldots & \ldots & \ldots \ldots \ldots & \ldots \ldots \\
x_{M, 1} & x_{M, 2} & x_{M, 3} & \ldots & x_{M, N}
\end{array}\right]
$$

where $x_{m, n}$ is the total number of CQI reports of value $n$ reported by the users in cell $\mathrm{m}$. This state space conveys two important factors of the network state to the contextual bandit: first, by observing the CQI reports, the quality of the users' channels can be considered, in particular, the number of users with good or bad channel conditions. Second, by collating the CQI reports from all users, it is also possible to observe the volume of users in a cell where $\sum_{n=1}^{N} x_{m, n}$ is proportional to the total number of users in cell $m$. In other words, if a cell $m$ has 3 associated users where two of the users return a wideband CQI value of 1 and the other user reports a wideband CQI value of 3 then the $m$ th row of state $X$ will be $\mathcal{X}_{m *}=\left[\begin{array}{lllll}2 & 0 & 1 & \ldots & 0\end{array}\right]$ which indicates that there are 2 users reporting CQI value of 1 and 1 user reporting CQI value of 3 .

However, conventional contextual bandit techniques do not scale well to large state spaces such as $\mathcal{X}$, as a result of having to visit every state action pair at least once. Instead, the agent is required to generalise over the large state space, by approximating a function that describes the relationship between the observed state of the environment and the predicted reward for taking each action. Neural networks are a popular method for function approximation in reinforcement learning, due to their computational simplicity and generality. The method proposed here uses a deep neural network to approximate this function. Specifically, a feed-forward network with two hidden layers is used, the input layer of the network observes the state of the environment as defined by $\mathcal{X}$ and calculates a prediction of the expected reward at the output $\mathcal{Y}$. The structure of this network can be seen in Fig. 1, where each output $y[i]$ corresponds to a joint scheduling policy $\sigma_{i}$.

The training process of neural networks for reinforcement learning is a widely explored area; Allesiardo et al. [25] describe the issues surrounding training neural networks for contextual multi-armed bandit problems. In particular, agents in reinforcement learning problems are more likely to take actions that are known to achieve good performance than those that do not. However, this can lead to the agent favouring actions that are taken more regularly. To counter this, Allesiardo et al. [25] describes methods for training contextual bandits: first, they consider that training the neural network over a large batch of training examples would improve the stability, as each action would be explored. Stochastic gradient descent is used to train the weights and biases of the network over stateaction-reward examples that the RL-DSS agent observes. The absolute error between the predicted reward and the received reward is defined by:

$$
E[i]= \begin{cases}y[i]-\mathcal{U}_{\sigma} & \text { if } \sigma_{i}=\sigma \\ 0 & \text { otherwise }\end{cases}
$$

where $E[i]$ is the absolute error at output with index $i, y[i]$ is the predicted reward at output with index $i$ and $\mathcal{U}_{\sigma}$ is the reward received by implementing joint scheduling policy $\sigma$. The loss function for the stochastic gradient descent is the mean absolute error.

However, with the batch training method, an extensive period of exploration is required to gather a large and varied 


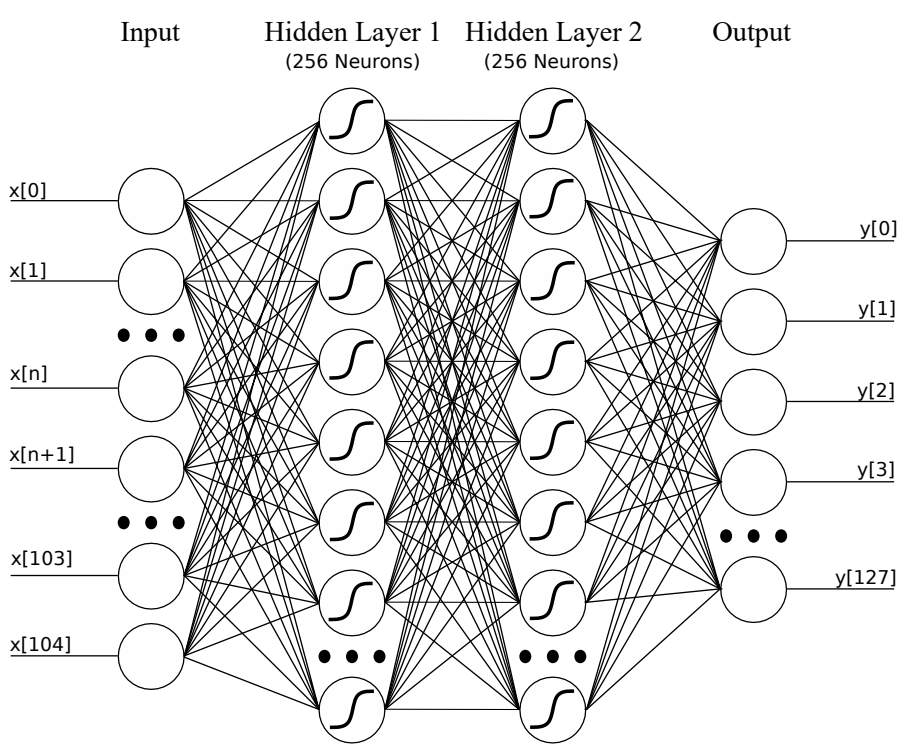

Fig. 1: The structure and parameters of the deep neural network used by the RL-DSS scheme. The network takes 105 inputs (15 CQI levels for each of the 7 cells) and has 128 outputs ( 7 cells each with 2 available schedulers). Two hidden feed forward layers consisting of 256 neurons are employed, each with a sigmoid activation function and an output layer with linear activation function.

training set that is then used to train the neural network. During this period the RL-DSS agent will produce random decisions that are likely to result in poor network performance. Further, the resulting agent would perform poorly on states outside of the initial training set, meaning retraining would need to take place if the conditions of the network were to change.

The alternative to batch training is online training, where the network is trained each time a decision is made. However, online training is problematic for deep reinforcement learners, as temporal correlation between states results in the learner favouring actions that are taken more regularly [26]. To prevent policy drift when training online, Allesiardo et al. [25] propose neuralbandit, a method that weights the loss associated with each reward by the probability of that action being selected by the agent. Under an $\epsilon$-greedy policy, where a uniform random action is taken with probability $\epsilon$ and the action with the best predicted reward is taken probability $1-\epsilon$, neuralbandit loss function can be defined as:

$$
E[i]= \begin{cases}\frac{y[i]-\mathcal{U}_{\sigma}}{\mathcal{P}(i)} & \text { if } \sigma_{i}=\sigma \\ 0 & \text { otherwise }\end{cases}
$$

where $\mathcal{P}(i)$ is the probability of selecting action $i$ given output $y[i]$, this can be expressed as:

$$
\mathcal{P}(i)= \begin{cases}1-\epsilon+\frac{\epsilon}{\mathcal{Y}} & \text { if } y[i]=\max _{i} y[i] \\ \frac{\epsilon}{\mathcal{Y}} & \text { otherwise }\end{cases}
$$

where $\mathcal{Y}$ is the number of outputs (number of possible actions).

Another well known method of stabilising the training process for deep reinforcement learning is experience replay [18], where the state-action-reward examples are stored in a replay

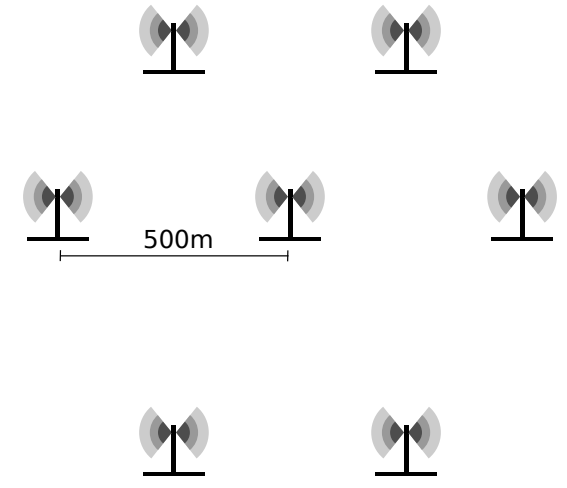

Fig. 2: Geographical distribution of cells for the network simulations.

memory as the agent is interacting with the environment. The neural network is then trained over a random batch of training examples from this memory. This method allows the learner to perform online learning while also making use of training examples that are not temporally correlated. Additionally, it allows the reinforcement learning method to utilise training data multiple times, resulting in a more efficient training process. For this study, we compare the performance of these three training methods for the RL-DSS problem using a simulation based evaluation.

\section{Simulated Evaluation}

To evaluate the performance of these schemes, a system level network simulation environment is used to simulate the performance of the network with the proposed schemes. We use a high volume of snap-shot simulations to train and test the performance of the DSS schemes.

In addition to the DSS schemes, static joint scheduling policies $\sigma=<0,0,0,0,0,0,0>$ and $\sigma=<1,1,1,1,1,1,1>$, referred to as $\sigma_{0}$ and $\sigma_{127}$ respectively from this point, are also simulated. We simulate $\sigma_{0}$ and $\sigma_{127}$ to generate a baseline to compare the DSS schemes against. The following sections will discuss the details of the simulation experiments performed and the parameters of the DSS systems that are evaluated.

\section{A. Simulation Environment}

The simulated network that consists of 7 cells in a hex grid layout; as shown in Fig2. Each cell has a channel bandwidth of $10 \mathrm{MHz}$, corresponding to a 50 physical resource blocks, with a carrier frequency of $2.14 \mathrm{GHz}$. There is a $500 \mathrm{~m}$ minimum separation between neighbouring cells and all cells have omnidirectional antennas with a maximum transmit power of $43 \mathrm{dBm}$. A large number of snap-shot scenarios are simulated over small periods (150 TTI) with static users.

To populate the simulated network with users, we use the user volume data recorded in the [12] data set. The data set contains 120 hourly data points where the user volumes are recorded for each individual cell. A group of 7 neighbouring cells is selected from the data set, corresponding to the 7 cells in the simulated network. User distribution scenarios are then generated for each cell in the simulated network. Users 


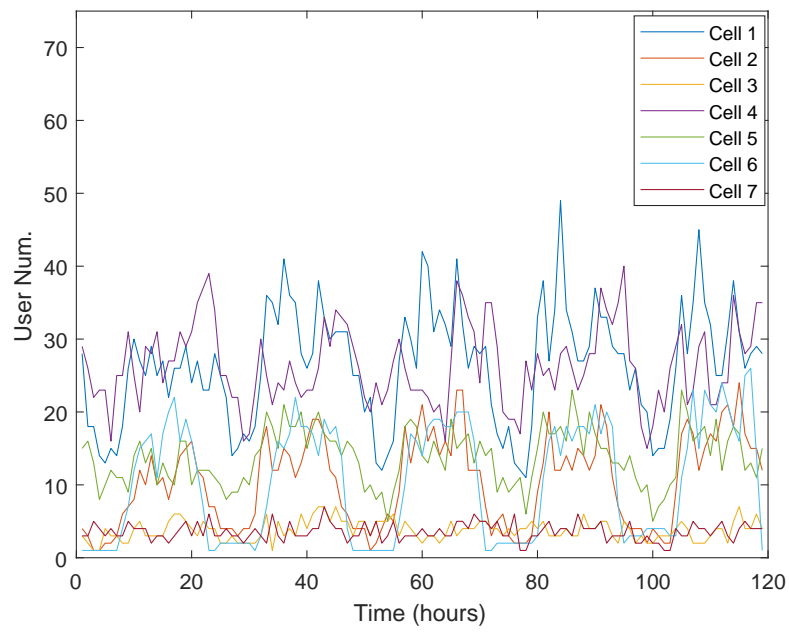

Fig. 3: The distribution of users over time for the selected neighbourhood of cells in the [12] test set.

TABLE I: Simulation Parameters

\begin{tabular}{|c|c|}
\hline Parameters & Value \\
\hline Number of Cells & 7 \\
\hline Carrier Frequency & $2.14 \mathrm{GHz}$ \\
\hline Channel Bandwidth & $10 \mathrm{MHz}$ \\
\hline Antenna Pattern & Omni-directional \\
\hline Pathloss Model & $\begin{array}{c}\text { Macro cell propagation model - Urban Area } \\
\text { (see 4.5.2 in }[27] \text { ) }\end{array}$ \\
\hline Traffic Model & Full Buffer \\
\hline Service Requirement & $1 \mathrm{Mbps}$ \\
\hline User Numbers & various \\
\hline User Distribution & uniform \\
\hline
\end{tabular}

are distributed uniformly within the coverage area of each simulated cell. The volume of users in each cell per hour can be seen in Fig 3 This process is performed for each cell and each hourly data point to create a library of scenarios that imitate the spatio-temporal distribution of users recorded in the data set. Each of the users in the network has a service requirement $T P^{(r e q)}=1$ Mbps.

This method is used to generate a test and training library of scenarios, consisting of 100 and 3000 scenarios for each hour in the data set for the test and training library respectively.

The simulation of large numbers of scenarios allows us to generate large volumes of training data for the learning methods that are used. Further, simulating large volumes of snapshot scenarios allows us to assess the long-term performance of these schemes, e.g. over the course of the week.

\section{B. Exploration Window}

The DSS schemes presented in this paper make joint scheduling policy decisions periodically where $\mathcal{T} e$ is the period between consecutive decisions. In order to find solutions to the DSS problem in a generic manner, both schemes are required to make decisions simply to explore the solution space. By their nature, exploratory decisions often result in a degradation in network performance, and it is therefore desirable to minimise the period for which these policies are implemented. However, due to the time-varying scheduling

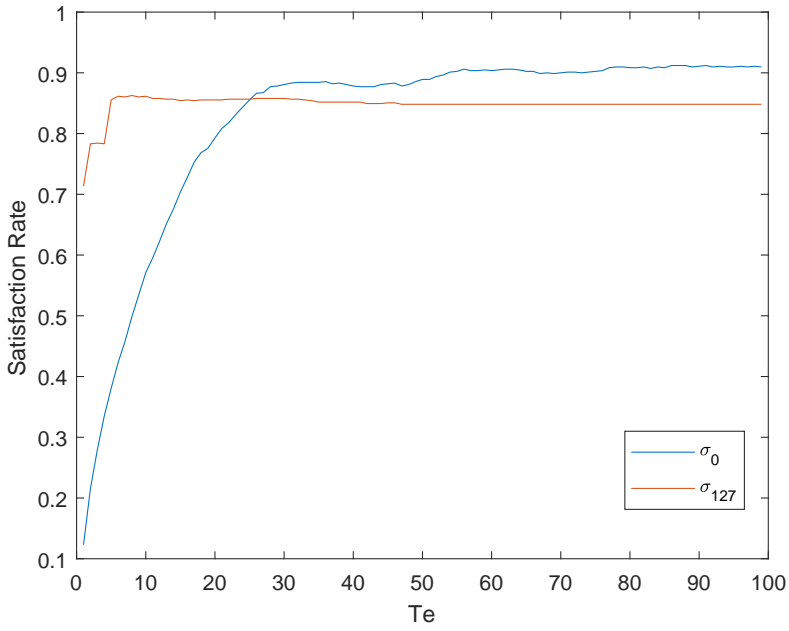

Fig. 4: Variation in the value of $\mathcal{U}_{\mathcal{T} e}$ (satisfaction rate) for $\sigma_{0}$ and $\sigma_{127}$ as $\mathcal{T} e$ increases from 1 to 100 TTI.

decisions made by schedulers, such as the proportional fair scheduler, even in static snap-shot simulations, the satisfaction rates recorded over small periods are not representative of the satisfaction rates achieved by the same policy over long periods of time. This is shown in Fig. 4 where the satisfaction rate for $\sigma_{0}$ and $\sigma_{127}$ are recorded over a large number or simulation scenarios as $\mathcal{T} e$ varies between 1 TTI and 100 TTI. To fulfil these conflicting criteria, $\mathcal{T} e$ must be set;

$$
\begin{array}{ll}
\text { minimize } & \mathcal{T} e \\
\text { subject to } & \left|\mathcal{U}_{\mathcal{T} e, \sigma}-\mathcal{U}_{T, \sigma}\right|<0.03
\end{array}
$$

where $\mathcal{U}_{\mathcal{T} e, \sigma}$ and $\mathcal{U}_{T, \sigma}$ are the satisfaction rates achieved by implementing policy $\sigma$ over implementation period $\mathcal{T} e$ and long time period $T$ (100s TTI) respectively.

For the snap-shot simulations performed here, it can be seen that $\mathcal{U}_{\mathcal{T} e, \sigma}$ achieved by $\sigma_{0}$ and $\sigma_{127}$ converge $90.96 \%$ and $84.82 \%$, respectively. For this study, we set $\mathcal{T} e=30$ TTI where $\mathcal{U}_{\mathcal{T} e, \sigma}$ of $\sigma_{0}$ and $\sigma_{127}$ are recorded at $88.07 \%$ and $85.78 \%$, respectively, deviating $2.89 \%$ and $0.96 \%$ from the converged values.

\section{DSS Parameters}

The genetic algorithm has a population that consists of 200 chromosomes and the mutation rate $\mu=0.01$. We simulate 60 generations of the genetic algorithm for each hourly training set, before the most popular policy from the 60th generation is selected and implemented for the entirety test set. The purpose of selecting the most popular policy at the conclusion of the exploration time is to prevent rapid changes in joint scheduling policy. To perform 60 generations of the genetic algorithm in with $\mathcal{T} e=30$ TTI takes 6 minutes. Therefore, repeating this training process once every hour uses approximately $10 \%$ of the total network time.

All three of the training methods for the RL-DSS entity are tested in this study. First, for the batch trained RL-DSS entity, a library of 100,000 training examples are randomly generated 
TABLE II: Satisfaction and error rates of the proposed schemes

\begin{tabular}{|c|c|c|}
\hline Scheduling Policy & Sat. Rate (\%) & Err. Rate (\%) \\
\hline$\sigma_{0}$ & 88.36 & \\
$\sigma_{127}$ & 77.08 & \\
Random & 79.45 & 40.01 \\
GA-DSS & 89.19 & 6.13 \\
RL-DSS (Batch) & 91.95 & 4.70 \\
RL-DSS (NeuralBandit) & 91.50 & 3.35 \\
RL-DSS (ER) & 90.55 & 3.03 \\
\hline
\end{tabular}

from the training set of scenarios. To generate these training examples, the DSS entity implements a uniform random policy over all of the training examples. After the examples are generated the DSS agent selects at random 256 examples from the library and performs stochastic gradient descent on the networks weights and biases. This process is repeated for each of the training epochs.

For the neuralbandit based RL-DSS entity, the agent is trained online as it takes actions. At the beginning of each period, the agent observes the state of the network and takes an action according to an $\epsilon$-greedy policy, then after a period of implementation, the agent receives a reward from the environment. The agent is then trained on its received reward according to the neuralbandit training method. At the beginning of the simulation experiment, the neural network is untrained, meaning it has no knowledge of the problem. To increase the amount of explorations performed during this early phase, $\epsilon=0.5$. After each 24 hour period; $\epsilon=\frac{\epsilon}{2}$ to a minimum of $\epsilon=0.005$ reducing the occurrence of explorations as the knowledge of the problem is increased.

Finally, for the experience replay training method, we generate a reply memory consisting of 1500 training examples generated from a 4 hour period in the network. Then, the agent proceeds to take decisions based on the observed network state. After receiving the reward from the network, the neural network is trained over a random batch of 128 training examples, including the state-action-reward example generated which was most recently received. After training, the examples from the agent's actions replace memories stored in the replay memory, where the new example has a uniform probability of replacing any of the training examples in the memory. The agent selects actions using an $\epsilon$-greedy policy with $\epsilon=0.5$ at the start of the experiment. After each 24 hour period; $\epsilon=\frac{\epsilon}{2}$ to a minimum of $\epsilon=0.005$ in the same way as the neuralbandit agent.

\section{DISCUSSION}

For each of the test scenarios generated, the network is simulated with GA-DSS and all 3 RL-DSS schemes as well as $\sigma_{0}$ and $\sigma_{127}$. The satisfaction rates of users is recorded during the simulation. Table $\Pi$ compares the satisfaction rate of each of the scheduler selection schemes over the entire test set.

First inspecting the outcome of the simulations on the static schemes, it can be seen that $\sigma_{0}$ achieves a better satisfaction rate and overall throughput than $\sigma_{127}$. The cause of this can be identified by analysing the user satisfaction rate individually for each of the hourly test sets, providing satisfaction rate as a

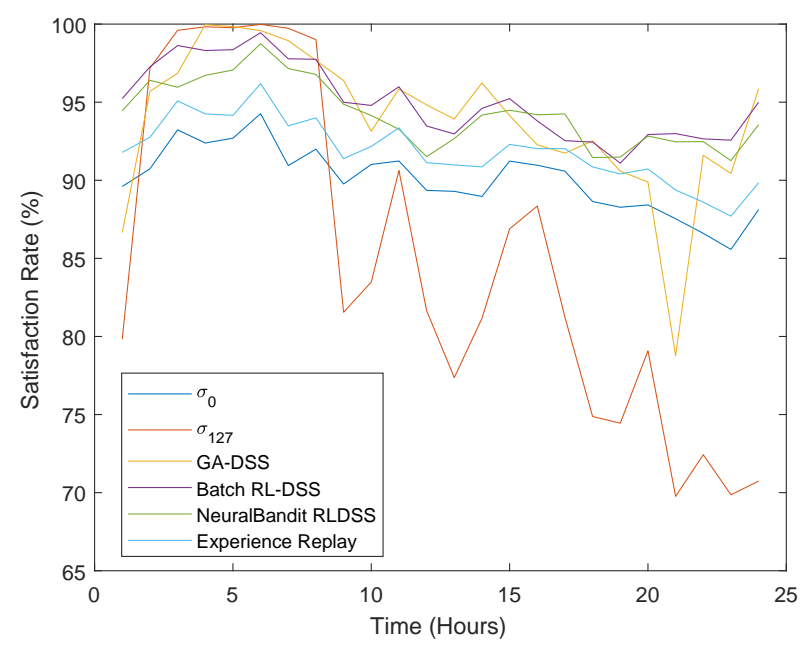

Fig. 5: Variation of satisfaction rate of the GA-DSS, trained RL-DSS (batch, neuralbandit and experience replay), $\sigma_{0}$ and $\sigma_{127}$ throughout the first 24 hours of the test set.

function of time. Upon close inspection of the first 24 hours of the test set, observed in Fig. 5. it can be seen that, for $\sigma_{127}$, the satisfaction rate approaches $100 \%$ between hours 3 and 7 when the constrained max-min scheduler can fulfil the satisfaction rates of the users in the network. Conversely, during the busiest scenarios, the performance of $\sigma_{127}$ degrades to a minimum of $69.54 \%$ for an hourly test set, when the scheduler is unable to fulfil the satisfaction rate of the users. For $\sigma_{0}$, the test data demonstrates a relative stability, maintaining an average satisfaction rate between $85.15 \%$ and $93.57 \%$ for the first 24 hours. The stability of the proportional fair scheduler makes the scheduler a popular choice for cellular networks. Despite its potentially high satisfaction rates, $\sigma_{127}$ is unsuitable for long term operation in networks such as those simulated in this study. As such, the potential benefits of employing this scheduler are likely to be overlooked in favour of the guaranteed performance of the proportional fair scheduler.

In Fig. 5 and Table $\Pi$ the advantages of dynamic scheduler selection can be seen. By selecting policies dynamically, the satisfaction rate over the entire test set can be improved when compared to the satisfaction rates of the static schemes. The overall satisfaction rate of the GA-DSS is recorded at $89.19 \%$. This value can be used as a benchmark to assess the effectiveness of the training methods employed by the RL-DSS schemes. For the batch, neuralbandit and experience replay training methods for the RL-DSS, the trained satisfaction rate over the entire test set is recorded at $91.95 \%, 91.50 \%$ and $90.55 \%$ respectively. All of the methods proposed improve the overall satisfaction rate of the users in the network when compared to the best performing static scheme $\left(\sigma_{0}\right)$. By inspecting which joint scheduling policy was chosen by the DSS systems, seen in Fig. 6, it can be seen that the increase in satisfaction rate is a result of implementing non-homogeneous joint scheduling policies and the ability to switch between policies based on the traffic in the network. Both neuralbandit and batch methods regularly switch the scheduling policies 

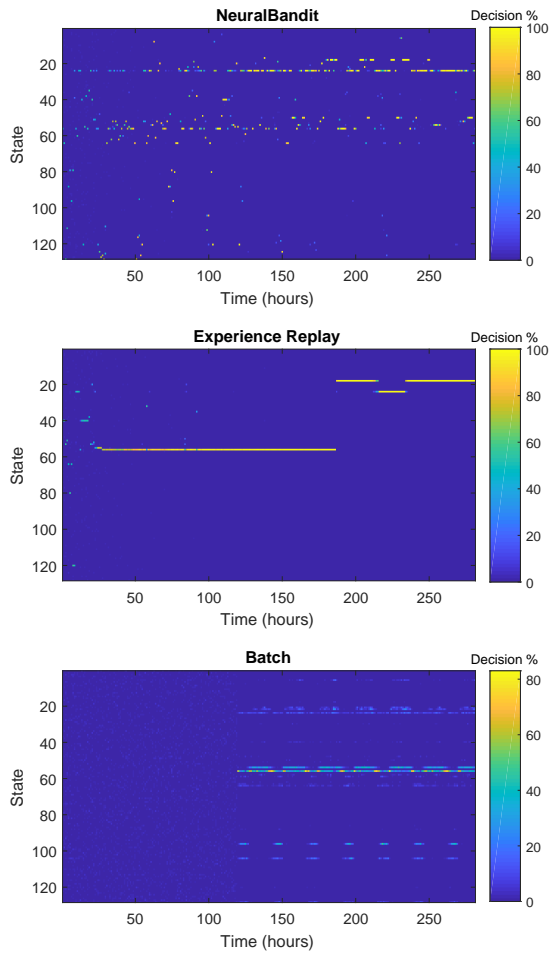

Fig. 6: Policies selected by the RL-DSS schemes throughout the simulation (state refers to the chosen joint scheduling policy $\left.\sigma_{\text {state }}\right)$.

to suit the network traffic. On the other hand, the experience replay method changes policy more infrequently (represented by the solid horizontal yellow lines).

By directly comparing each individual test scenario under different scheduler selection schemes, it can be determined which scheme performed best in each of the scenarios in the test sets. From this data, it can be established how accurate the selection methods are at selecting policies that achieve a performance increase against $\sigma_{0}$ and $\sigma_{127}$. This analysis is performed over the entire test set for each of the selection schemes.

First, inspecting the peak weekly performance of the GADSS scheme, it is established that the policy selected by GADSS outperforms the static policy $\sigma_{0}$ in $87.17 \%$ of the test scenarios and both $\sigma_{0}$ and $\sigma_{127}$ in $68.08 \%$ of the test scenarios. Comparing this to the RL-DSS schemes we can see that for the batch, neuralbandit and experience replay trained RLDSS schemes, the policies selected outperform $\sigma_{0}$ in $82.23 \%$, $90.66 \%$ and $88.90 \%$ of the test scenarios respectively and both $\sigma_{0}$ and $\sigma_{127}$ in $69.48 \%, 72.50 \%$ and $70.82 \%$ of the test scenarios respectively.

However, one of the factors that will impact most heavily on the viability of DSS schemes is the frequency and severity of errors in decision making. An erroneous decision is a decision that results in unacceptable network performance. For this study, erroneous decisions are identified by comparing each test case with the lowest satisfaction rate achieved by $\sigma_{0}$ in the same hourly test set. This measure identifies decisions that lead to satisfaction rates outside of the range of the standard scheduler deployment. The rate at which these erroneous decisions are made is defined as the error rate. For the GADSS $6.13 \%$ of the decisions made by the agent where classed as erroneous, while for the trained RL-DSS methods $4.70 \%$, $3.35 \%$ and $3.03 \%$ of the decisions made were considered erroneous.

The GA-DSS scheme and the batch training method are both able to consistently select schemes more often that are most beneficial to the user satisfaction rate. However, both of these schemes are also more prone to making erroneous decisions that result in poor user satisfaction. Additionally, as per [25] the batch training method is unlikely to operate well if the network conditions were to change. This method, therefore, is reliant on the traffic patterns observed repeating on a weekly basis, negating the need for regular re-training. On the other hand, the neuralbandit and experience reply methods both improve the overall satisfaction rate of users in the network, but do so with reduced erroneous decision rates. Further, the online nature of training allows the RL-DSS scheme to react to changes in network traffic week on week.

\section{A. Genetic Algorithm}

The GA-DSS scheme relies on the temporal correlation of network states to select a suitable joint scheduling policy. Without the ability to base its decisions on contextual information, the GA-DSS scheme is required to run the genetic algorithm every time the network undergoes significant changes. As the test examples in this study are divided amongst hourly test sets, the GA-DSS scheme undergoes this exploration every hour. The effect this exploration has on the satisfaction rate can be seen in Fig. 7. It can be seen that the satisfaction rates of users in the network regularly drop significantly at the beginning of the exploration phases. The selection policy chooses the most common joint scheduling policy in the final generation, once exploration finishes. This selection policy does not always match the satisfaction rate achieved at the end of exploration.

\section{B. Reinforcement Learning}

When training via online reinforcement learning methods such as neuralbandit and experience replay, the quality of the decisions made is expected to increase over the period of training. To assess the impact of this training, the error rate and accuracy of the decisions made by the DSS schemes are recorded, including the random decisions made for exploration purposes. This can be seen in Fig 8 and Fig 9

In the first 5-day cycle, all three methods of training for RLDSS require large amounts of exploration. The batch training method implements random policies for the entirety of the first 5-day cycle, resulting in low accuracy (29.39\%) and a large 


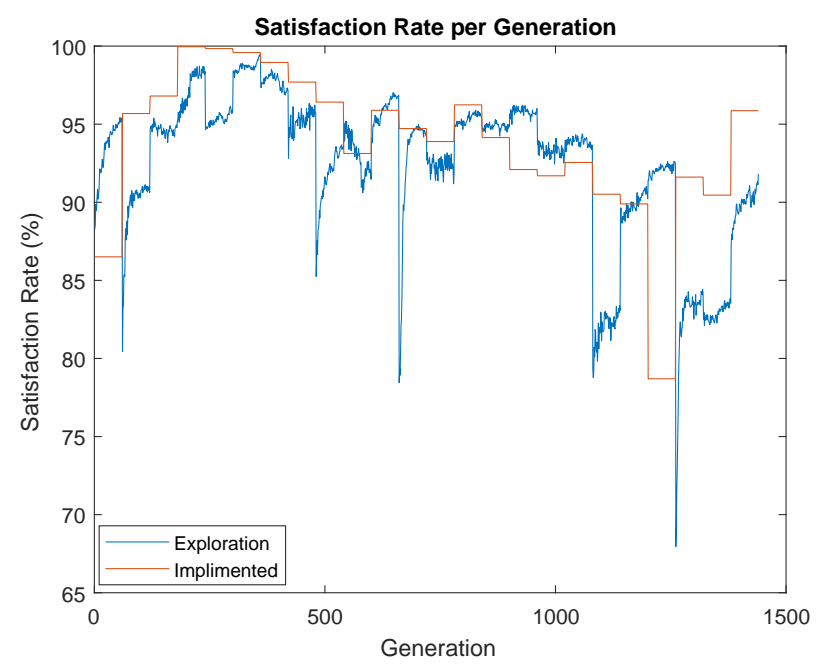

Fig. 7: Variation of satisfaction rate as the genetic algorithm runs through generations as well as the satisfaction rates of the policy implemented at the conclusion of the exploration period.

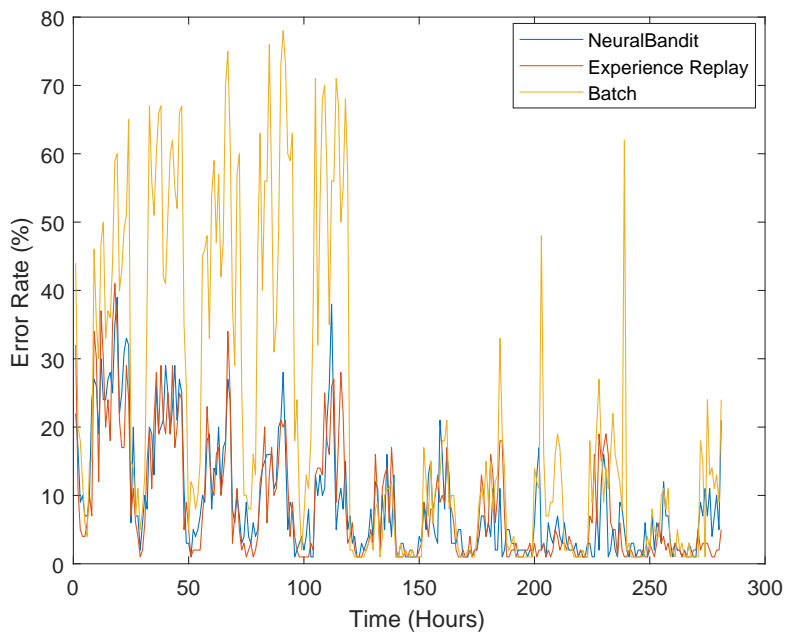

Fig. 8: Percentage of erroneous decisions made by the DSS scheme throughout the training process.

error rate $(40.01 \%)$. The online training methods are able to improve their policy selection within hours of starting training. The result is that the accuracy and error rate of the methods in the first 5-days are much improved against the batch method, with neuralbandit achieving an accuracy of $68.48 \%$ and an error rate of $14.67 \%$. Experience replay achieves an accuracy of $64.06 \%$ and an error rate of $12.90 \%$. The effect this training has on the overall satisfaction rates of the network can also be observed, with the batch methods achieving a $79.45 \%$ satisfaction rate, the neuralbandit a $89.04 \%$ satisfaction rate, and experience replay a $89.50 \%$ satisfaction rate.

By the second 5-day period, the RL-DSS methods have undergone significant training, allowing the selection of better policies; this results in improved error rates for all of the methods. The batch method is able to achieves an error

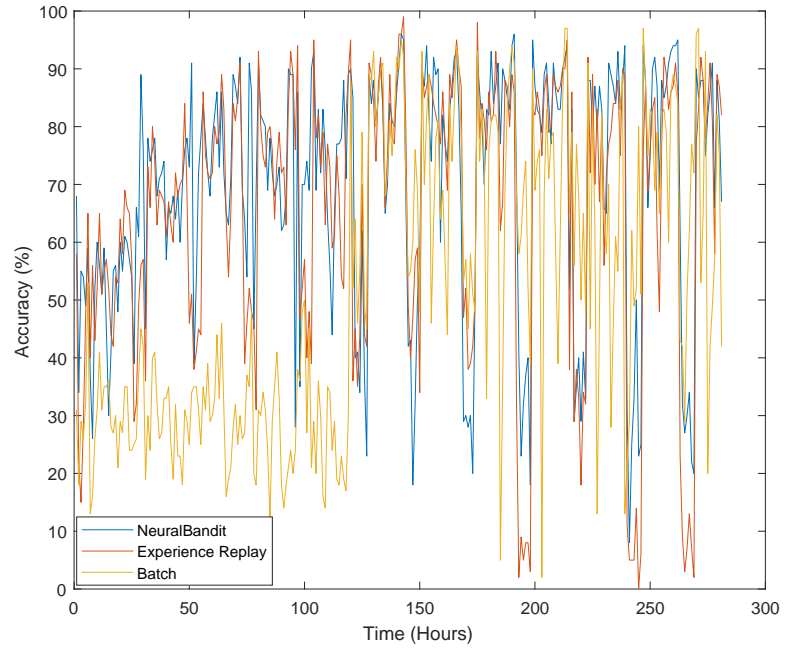

Fig. 9: Accuracy of decisions made by the DSS scheme throughout the training process.

rate of $4.70 \%$, while the neuralbandit and experience replay methods are able to achieve $3.97 \%$ and $4.41 \%$ respectively. However, the online training methods do not dramatically increase the accuracy of their selection policies; the accuracy of neuralbandit for the second 5-day period is $72.50 \%$, while the accuracy of the experience replay method is $70.82 \%$.

\section{CONCLUSION}

In this study, we present two methods for performing dynamic scheduler selection. One of these schemes is based on a nature-inspired genetic algorithm, while the other utilises deep reinforcement learning methods. Network simulations show that both methods are able to make decisions that utilise the available schedulers to increase the satisfaction rate of the cellular network users against the conventional static scheduler deployment. Reinforcement learning based methods require a large and diverse training set gathered over many hours in order for decisions to show significant improvement. However, after this training set is gathered, reinforcement learning methods can make instantaneous decisions that improve the satisfaction rate of users. Conversely, the genetic algorithm can explore and converge on a solution over a smaller time period. However, this period of exploration is required to be performed constantly, resulting in periods of reduced satisfaction rate when the network conditions are significantly altered. Further, once trained, the reinforcement learning based systems are able to achieve greater gains to satisfaction rate and make fewer erroneous decisions. This makes the reinforcement learning methods better suited to long term use.

The training method for reinforcement learning has an important impact on the performance of the dynamic scheduler selection scheme; training over large batches of random experience generated over a large time scale results in the best overall satisfaction rates. However, this method is also more prone to making erroneous decisions that result in performance which does not meet expectations. Further, this method of 
training relies on user patterns repeating over a large period of time. Changes in user distributions in the network are likely to require retraining the RL-DSS scheme over a batch of experience, including the new user distributions.

Conversely, the online training methods achieve a more modest improvement to the overall satisfaction rates in the network. However, the method's training process allows the entity to make beneficial decisions more quickly. These methods are also more reactive to changing user distributions, meaning retraining does not have to occur if the user distribution changes significantly.

The reinforcement learning based dynamic scheduler selection schemes were designed to be generic, allowing implementation with multiple different types of scheduler. Such a system allows for unplanned distribution of scheduling methods, facilitating dense multi-vendor networks without the need to define new inter-scheduler communication standards or standardised scheduling. The next steps towards generic scheduler control using a dynamic scheduler selection scheme will involve increasing the scalability of these techniques. The number of actions in the DSS problem scales exponentially with the number of cells in the network. The exponential relationship between the number of actions and the number of cells means that performing DSS in the methods defined here over larger clusters scales badly. Further development of these methods should look to improve the scalability. Additionally, complimentary techniques to classify dense networks into manageable cluster sizes can be implemented to control the scale of these action spaces.

\section{REFERENCES}

[1] F. Capozzi, G. Piro, L. A. Grieco, G. Boggia, and P. Camarda, "Downlink packet scheduling in lte cellular networks: Key design issues and a survey," IEEE communications surveys \& tutorials, vol. 15, no. 2, pp. 678-700, 2012.

[2] S. F. Sulthana and R. Nakkeeran, "Study of downlink scheduling algorithms in lte networks," Journal of Networks, vol. 9, no. 12, p. 3381, 2014.

[3] E. Hossain, M. Rasti, H. Tabassum, and A. Abdelnasser, "Evolution towards 5g multi-tier cellular wireless networks: An interference management perspective," arXiv preprint arXiv:1401.5530, 2014.

[4] 3rd Generation Partnership Project; Technical Specification Group Radio Access Network, "3GPP TR 38.801 Study on New Radio Access Technology; Radio Access Architecture and Interfaces," Tech. Rep. Release 14, 2017.

[5] A. Douik, H. Dahrouj, T. Y. Al-Naffouri, and M.-S. Alouini, "Coordinated scheduling for the downlink of cloud radio-access networks," in 2015 IEEE International Conference on Communications (ICC). IEEE, 2015, pp. 2906-2911.

[6] G. Nardini, G. Stea, A. Virdis, A. Frangioni, L. Galli, D. Sabella, and G. Dell'Aera, "Practical feasibility, scalability and effectiveness of coordinated scheduling algorithms in cellular networks towards $5 \mathrm{~g}$," Journal of Network and Computer Applications, vol. 106, pp. 1-16, 2018.

[7] O. D. Ramos-Cantor and M. Pesavento, "Decentralized coordinated scheduling with muting in lte-advanced networks," in 2017 IEEE 18th International Workshop on Signal Processing Advances in Wireless Communications (SPAWC). IEEE, 2017, pp. 1-5.

[8] D. López-Pérez, A. Ladányi, A. Jüttner, H. Rivano, and J. Zhang, "Optimization method for the joint allocation of modulation schemes, coding rates, resource blocks and power in self-organizing lte networks," in 2011 Proceedings IEEE INFOCOM. IEEE, 2011, pp. 111-115.

[9] A. U. Ahmed, M. T. Islam, and M. Ismail, "A review on femtocell and its diverse interference mitigation techniques in heterogeneous network," Wireless personal communications, vol. 78, no. 1, pp. 85-106, 2014.
[10] A. L. Stolyar and H. Viswanathan, "Self-organizing dynamic fractional frequency reuse in ofdma systems." in Infocom, 2008, pp. 691-699.

[11] D. Lopez-Perez, X. Chu, A. V. Vasilakos, and H. Claussen, "Power minimization based resource allocation for interference mitigation in ofdma femtocell networks," IEEE Journal on Selected Areas in Communications, vol. 32, no. 2, pp. 333-344, 2013.

[12] X. Chen, Y. Jin, S. Qiang, W. Hu, and K. Jiang, "Analyzing and modeling spatio-temporal dependence of cellular traffic at city scale," in 2015 IEEE International Conference on Communications (ICC). IEEE, 2015, pp. 3585-3591.

[13] A. Zakrzewska, S. Ruepp, and M. S. Berger, "Towards converged 5g mobile networks-challenges and current trends," in Proceedings of the 2014 ITU kaleidoscope academic conference: Living in a converged world-Impossible without standards? IEEE, 2014, pp. 39-45.

[14] A. Imran, A. Zoha, and A. Abu-Dayya, "Challenges in 5g: how to empower son with big data for enabling 5g," IEEE network, vol. 28 , no. 6 , pp. $27-33,2014$.

[15] "O-ran: Towards an open and smart ran," O-RAN Alliance, Tech. Rep., oct 2018.

[16] P. V. Klaine, M. A. Imran, O. Onireti, and R. D. Souza, "A survey of machine learning techniques applied to self-organizing cellular networks," IEEE Communications Surveys \& Tutorials, vol. 19, no. 4, pp. 2392-2431, 2017.

[17] M. Jaber, M. Imran, R. Tafazolli, and A. Tukmanov, "An adaptive backhaul-aware cell range extension approach," in 2015 IEEE International Conference on Communication Workshop (ICCW). IEEE, 2015, pp. 74-79.

[18] V. Mnih, K. Kavukcuoglu, D. Silver, A. Graves, I. Antonoglou, D. Wierstra, and M. Riedmiller, "Playing atari with deep reinforcement learning," arXiv preprint arXiv:1312.5602, 2013.

[19] S. Chinchali, P. Hu, T. Chu, M. Sharma, M. Bansal, R. Misra, M. Pavone, and S. Katti, "Cellular network traffic scheduling with deep reinforcement learning," in Thirty-Second AAAI Conference on Artificial Intelligence, 2018.

[20] C. Zhang, P. Patras, and H. Haddadi, "Deep learning in mobile and wireless networking: A survey," IEEE Communications Surveys \& Tutorials, 2019.

[21] I.-S. Comşa, S. Zhang, M. E. Aydin, P. Kuonen, Y. Lu, R. Trestian, and G. Ghinea, "Towards 5g: A reinforcement learning-based scheduling solution for data traffic management," IEEE Transactions on Network and Service Management, vol. 15, no. 4, pp. 1661-1675, 2018.

[22] R. Kwan, C. Leung, and J. Zhang, "Proportional fair multiuser scheduling in lte," IEEE Signal Processing Letters, vol. 16, no. 6, pp. 461-464, 2009.

[23] Y. Li, M. Sheng, C. W. Tan, Y. Zhang, Y. Sun, X. Wang, Y. Shi, and J. Li, "Energy-efficient subcarrier assignment and power allocation in ofdma systems with max-min fairness guarantees," IEEE Transactions on Communications, vol. 63, no. 9, pp. 3183-3195, 2015.

[24] 3rd Generation Partnership Project; Technical Specification Group Radio Access Network, "3GPP TS 36.213 Evolved Universal Terrestrial Radio Access (E-UTRA); Physical layer procedures," Tech. Rep. Release 15, 2019.

[25] R. Allesiardo, R. Féraud, and D. Bouneffouf, "A neural networks committee for the contextual bandit problem," in International Conference on Neural Information Processing. Springer, 2014, pp. 374-381.

[26] J. N. Tsitsiklis and B. Van Roy, "Analysis of temporal-difference learning with function approximation," in Advances in neural information processing systems, 1997, pp. 1075-1081.

[27] 3rd Generation Partnership Project; Technical Specification Group Radio Access Network;, "3gpp tr 36.942 evolved universal terrestrial radio access (e-utra); radio frequency (rf) system scenarioss," Tech. Rep. Release 15, 2018 